\title{
Identification of a novel frameshift mutation of the $E X T 2$ gene in a family with multiple osteochondroma
}

\author{
PENG XIA ${ }^{1}$, HAIKUN XU ${ }^{2}$, QINGYANG SHI ${ }^{3}$ and DEJUN LI ${ }^{3}$ \\ ${ }^{1}$ Department of Spinal Surgery, Orthopedics Hospital, The Second Hospital of Jilin University, Changchun, Jilin 130041; \\ ${ }^{2}$ Department of Cardiology, China-Japan Union Hospital of Jilin University, Changchun, Jilin 130033; \\ ${ }^{3}$ Center for Prenatal Diagnosis, The First Hospital of Jilin University, Changchun, Jilin 130021, P.R. China
}

Received November 16, 2014; Accepted October 2, 2015

DOI: $10.3892 / \mathrm{ol} .2015 .3844$

\begin{abstract}
Multiple osteochondroma (MO), also known as multiple hereditary exostoses, is an autosomal dominant skeletal disorder with characteristic multiple cartilage-capped tumours (osteochondromas or exostoses) growing outward from the metaphyseal region of the long tubular bones. Mutations in exostosin glycosyltransferase 1 (EXT1) or EXT2 are the most commonly associated mutations with $\mathrm{MO}$ and are responsible for $70-95 \%$ of cases. In the present study, a genetic analysis was performed on a large family with MO using polymerase chain reaction and direct DNA sequencing of the entire coding regions of EXT1 and EXT2. Sanger sequencing identified a novel heterozygous frameshift mutation, c.119_120delCT (p.Thr40ArgfsX15), in exon 2 of the EXT2 gene in the proband and all other affected individuals, while this deleterious mutation was not detected in the healthy family members and normal controls. The c.119_120delCT mutation is located in the transmembrane region of the EXT2 protein and results in a truncated EXT2 protein lacking 665 amino acids at the $\mathrm{C}$-terminus, which includes the critical exostosin and glycosyltransferase family 64 domains. Thus, the present study identified a novel causative frameshift mutation in EXT2 from a large MO family. This study is useful for extending the known mutational spectrum of EXT2, for understanding the genetic basis of MO in the patients studied, and for further application of mutation screening in the genetic counseling and subsequent prenatal diagnosis of this family.
\end{abstract}

\section{Introduction}

Multiple osteochondroma (MO; MIM \#133700 and \#133701), also known as multiple hereditary exostoses, is an autosomal dominant skeletal disorder with characteristic multiple

Correspondence to: Dr Dejun Li, Center for Prenatal Diagnosis, The First Hospital of Jilin University, 71 Xinmin Street, Chaoyang, Changchun, Jilin 130021, P.R. China

E-mail: lidejun@jlu.edu.cn

Key words: multiple osteochondroma, EXT2, frameshift mutation cartilage-capped tumours (exostoses or osteochondromas) growing outward from the metaphyseal region of the long tubular bones. The estimated prevalence of MO has been recorded to range from $1 / 100$ in a small Guam population (1) to $1.3 / 100,000$ in a European population (2) and at least $1 / 50,000$ in a Western population (3). Osteochondromas are a consequence of the superfluous proliferation of chondrocytes and bone growth at the juxta-epiphyseal regions of the long bones, and are the most frequently identified benign bone tumour. Osteochondromas are slow-growing during the first decades of life, growing in number and size until the closure of the growth plates upon skeletal maturation at the end of puberty. Characteristic features of MO consist of significant inter- and intra-familial phenotypic variability, including variability in the size and number of the osteochondromas, the location and number of the associated bones, and the degree of the deformity. Although benign, osteochondromas can result in a number of secondary complications, including compression of the blood vessels, tendons and nerves. Individuals with osteochondroma are often of short stature, with deformities leading to problems in functionality. The most serious secondary complication is the malignant transformation toward a secondary peripheral chondrosarcoma, which occurs in $0.5-5 \%$ of cases (4).

$\mathrm{MO}$ is a genetically heterogeneous disease. At least 2 loci have been isolated by linkage analysis and positional cloning: EXT1 (MIM \#608177) maps to chromosome 8q24.11-q24.13, spans $350 \mathrm{~kb}$ and is comprised of 11 exons (5), while EXT2 (MIM \#608210) maps to chromosome 11p11-p11.2, spans almost $108 \mathrm{~kb}$ and is comprised of 16 exons (6). EXT1 and EXT2 belong to the putative tumour-suppressor EXT gene family, which also contains three homologous EXT-like (EXTL) genes: EXTL1, EXTL2 and EXTL3 (7-9). All EXT gene family members share a homologous carboxyl terminus and encode glycosyltransferases that are involved in heparin sulfate (HS) biosynthesis (10). A number of studies have investigated MO-causing mutations in a variety of populations (11-13). These data are being gathered in the Multiple Osteochondromas Mutation Database (http://medgen.ua.ac. be/LOVDv.2.0/). To date, $>400$ and 200 mutations have been identified in EXT1 and EXT2, respectively. The majority of the mutations (80\%) are nonsense, frameshift and splice site mutations, which lead to the premature termination of translation, or are involved in the partial or total deletion of the gene (14). 
The present study screened for mutations in the EXT1/EXT2 genes using polymerase chain reaction (PCR) and direct sequencing in three generations of a family with $\mathrm{MO}$. A novel frameshift mutation, c.119_120delCT (p.Thr40ArgfsX15), was consequently identified in exon 2 of the EXT2 gene.

\section{Patients and methods}

Patients. Three generations of a MO family from Jilin, China, were investigated in the present study (Fig. 1). From the family, 8 members were affected with MO, including 2 deceased individuals. The proband (III-16) was a 28 -year-old man suffering from multiple exostoses involving the bilateral proximal humeri, the left ulnar, the bilateral proximal and distal tibiae, the bilateral distal femurs and the right proximal femur (Fig. 2). On January 13, 2014, the proband underwent osteochondroma surgery for the right femur at Country Hospital of Fusong (Fusong, China) due to pain and functional limitations. At 15 days post-surgery (January 28, 2014), the proband suffered a subtrochanteric fracture of the right femur and was referred to the Second Hospital of Jilin University (Changchun, China), where internal fixation of the fracture site was performed.

Clinical data of each subject was recorded by nurse-administered questionnaires. Each participant underwent careful physical and/or radiographic examinations by two or more experienced experts at the Department of Orthopedics, The Second Hospital of Jilin University (Changchun, Jilin, China). $\mathrm{MO}$ was classified as at least 2 osteochondromas, arising from the lateral ends of the humeri, ulnae, femurs, tibiae, fibulae or knee joints. A group of 50 unrelated healthy subjects, matched for geographical ancestry, were included as controls. EDTA-anticoagulated peripheral blood $(5 \mathrm{ml})$ was drawn from 10 family members (Table I) and from the 50 healthy controls. Written informed consent was obtained from all subjects or their respective guardians prior to their participation in the experimental protocol. This study was approved by the Ethics Review Committee of the Second Hospital of Jilin University.

Genetic analysis. Genomic DNA was extracted from peripheral blood leukocytes using the QIAamp DNA Blood Mini kit (Qiagen, Hilden, Germany) according to the manufacturer's protocols. All exons and flanking intronic regions of the EXTI and EXT2 genes were amplified by PCR using the primers listed in Table II. The amplifications were performed to a final volume of $50 \mu \mathrm{l}$, containing $1 \mu \mathrm{l}$ genomic DNA, $25 \mu \mathrm{l} 2 \mathrm{X}$ Premix Taq ${ }^{\mathrm{TM}}$ (Takara Biotechnology Co., Ltd., Dalian, China), $1 \mu \mathrm{l}$ of each primer and $22 \mu \mathrm{l}$ sterilized distilled water. All PCR programs included an initial denaturation of $5 \mathrm{~min}$ at $95^{\circ} \mathrm{C}$, followed by 35 cycles of $30 \mathrm{sec}$ at $95^{\circ} \mathrm{C}, 45 \mathrm{sec}$ at annealing temperature (Ta) and $30 \mathrm{sec}$ at $72^{\circ} \mathrm{C}$. Finally, an extension at $72^{\circ} \mathrm{C}$ was performed for $10 \mathrm{~min}$. Ta was $60^{\circ} \mathrm{C}$ for all primer combinations, with the exception of primers for the amplification of overlapping regions of exon 1 of EXTl. For these primer combinations, Ta was set at $57^{\circ} \mathrm{C}$ for ex1-2 and ex1-3. Direct sequencing of the PCR products was performed using BigDye ${ }^{\circledR}$ Terminator v3.1 (Applied Biosystems, Foster City, CA, USA) with the ABI 3730 automated sequencer. Sequences generated from patients were compared with the published EXT1 and EXT2 reference sequences from the National Center for Biotechnology Information (http://www.ncbi.nlm.nih.gov/; GenBank accession
Table I. Characteristics of study subjects in the MO family.

\begin{tabular}{lccc}
\hline Subject & Age, years & Gender & MO status \\
\hline II-5 & 62 & Female & Unaffected \\
II-7 & 61 & Male & Affected \\
II-17 & 48 & Female & Affected \\
III-9 & 37 & Male & Affected \\
III-10 & 33 & Male & Affected \\
III-11 & 30 & Female & Unaffected \\
III-14 & 29 & Male & Unaffected \\
III-16 & 28 & Male & Affected \\
III-17 & 24 & Male & Unaffected \\
III-18 & 25 & Male & Affected
\end{tabular}

MO, multiple osteochondroma.

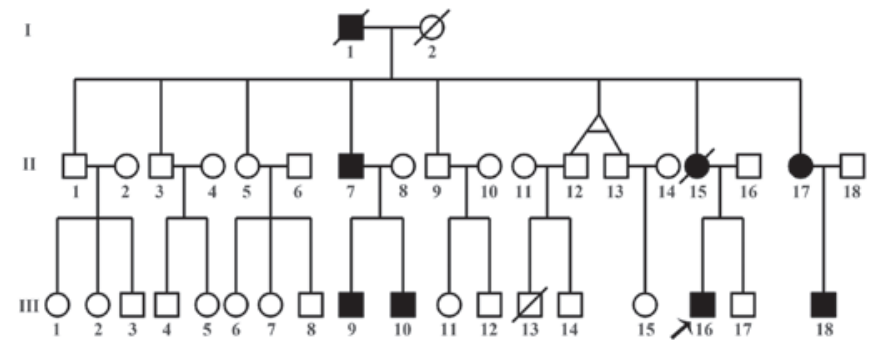

Figure 1. Pedigree of the family with multiple osteochondroma. The proband (III-16) is noted with an arrow. $\mathbf{n}$, affected male; $\bullet$, affected female; $\square$, healthy male; $\bigcirc$, healthy female. Boxes or circles with an oblique line mean that the individual is deceased.

nos. NM_000127.2 and NM_207122.1, respectively). When an insertion/deletion mutation was found, the amplified products with mutations were first ligated to a pMD-18T vector (Takara Biotechnology Co., Ltd.) and transformed into Escherichia coli. Next, sequencing was performed with plasmids extracted from E. coli using the AxyPrep Plasmid Miniprep kit (Axygen Biosciences, Union City, CA, USA). In addition, detected mutations were confirmed in another 50 unrelated healthy controls.

In silico analysis and homology study. When a mutation that had never been reported was found, the functional consequences of amino acid changes were predicted using the Mutation Taster software (http://www.mutationtaster.org). Regarding the homology study, ClustalW2 software (http://www.ebi.ac.uk/Tools/msa/clustalw2/) was used to compare the human EXT2 protein sequence (NP_997005.1) with the homologs from Pan troglodytes, Macaca mulatta, Mus musculus, Rattus norvegicus, Canis lupus familiaris, Bos taurus, Gallus gallus, Danio rerio and Xenopus (sequences acquired from http://www.ensembl.org/).

\section{Results}

Genetic analysis identifies a novel frameshift mutation (c.119_120delCT) in EXT2 as the causative mutation in the MO family. Following sequencing of all the exons and 
Table II. Primers used to amplify the exons of the EXT1 and EXT2 genes.

\begin{tabular}{|c|c|c|c|c|c|}
\hline Gene & Exon & Upstream primer $\left(5^{\prime}-3^{\prime}\right)$ & Downstream primer $\left(5^{\prime}-3^{\prime}\right)$ & $\begin{array}{l}\text { Amplicon } \\
\text { length, bp }\end{array}$ & $\mathrm{Ta},{ }^{\circ} \mathrm{C}$ \\
\hline \multirow[t]{13}{*}{ EXT1 } & Ex1-1 ${ }^{\mathrm{a}}$ & GGAGAGTTTGAAGTCTTTACAGGC & TGGGGTCCGAGGTGTAGAAC & 561 & 60 \\
\hline & $\operatorname{Ex} 1-2^{a}$ & GGCTTGCAGTTTAGGGCATCGAG & TTGTTCCACAAGTGGAGACTCTG & 485 & 57 \\
\hline & Ex $1-3^{a}$ & CCAGTTGTCACCTCAGTATGTGC & AACTTCACACCTGGACCAAG & 543 & 57 \\
\hline & Ex2 & TGCGTAAATTCATGCACATGG & GGAGAGGTGATAATGTTAAACC & 268 & 60 \\
\hline & Ex3 & TGCCAGTCATTGAGTTTGTAC & GGAGATTTTGTTGGAAAGTGAA & 386 & 60 \\
\hline & Ex4 & CTATATGCTAGAAGCCAAATGC & CAATATATCCAAGTACAGGAATC & 372 & 60 \\
\hline & Ex5 & TCCATTAСТСТСТСТGTCTTG & ATGCAGGGTGTTAGATGGAC & 410 & 60 \\
\hline & Ex6 & CCTGTCAGGACATAAGAAGC & TGAAAAGGGTGTAACGAGGC & 426 & 60 \\
\hline & Ex7 & TCTGCCGTTTTGTCTTGCTG & ATACACACAAGGTCACAAAGC & 450 & 60 \\
\hline & Ex8 & GTGAGGATGGGAGAATTGTC & AGGAATCGGGCTGATTAAAAC & 334 & 60 \\
\hline & Ex9 & GAATTAATGTTTCGCCACAGTC & AAACACACATTTGACACATCAG & 435 & 60 \\
\hline & Ex10 & CATCATCATTATCATTAССАТТC & GGAGAGCTTTACATCCTTGG & 525 & 60 \\
\hline & Ex11 & TTGCTGCTTGCTCATTTGCC & TTTGTCATTCTGCTCATCTAAG & 420 & 60 \\
\hline \multirow[t]{14}{*}{ EXT2 } & Ex2-1 ${ }^{\mathrm{a}}$ & TGAGTGACAGAGTGAAACCC & CAGAGCATAGATATACACCTTG & 488 & 60 \\
\hline & $\operatorname{Ex} 2-2^{a}$ & AGCCGACAGTCCCATCCC & AAACCAACTCAAGAGCAGAAG & 425 & 60 \\
\hline & Ex3 & TGGGATTTCCAGGAGTTTGC & САСТTСТАAАТСТTCAGGAGG & 390 & 60 \\
\hline & Ex4 & AGCAGAGAGGCTGTCCGTAA & ATAGGAAGCCGTTTCAGCAA & 805 & 60 \\
\hline & Ex5 & AGGGACTCAGATGTAACTAAGA & ACGAACACAAGACACCAGAC & 792 & 60 \\
\hline & Ex6 & AGTATTGCTTGGCGTCAACC & TAGACCAGTGTACTAACTCTC & 401 & 60 \\
\hline & Ex7 & AATGGAGCTGTAAGAGAACTC & ATCTAGTGGAGGAAGTAAACC & 408 & 60 \\
\hline & Ex8 & TGGCTTGAACAGCAGGGAG & AATTATGCTGCCCTTATCAGG & 446 & 60 \\
\hline & Ex9 & CACCAAGCCTGCCATGTTTG & GGCATGCTGTCTCAGAAATG & 408 & 60 \\
\hline & Ex10 & ACCTTTGGATTTGATGAGAGC & TAACCCACACTCTTACGCAC & 450 & 60 \\
\hline & Ex11 & TCTCCAGAATCCCATTATGAC & CATATTTTCTACTATGAGCGTG & 469 & 60 \\
\hline & Ex12 & GTCACTTGACCAAAAGCATTC & GAGCTTAAAGTTTATCTAGTCC & 417 & 60 \\
\hline & Ex13 & CTTGTGAGTTCTGCCGTTGG & ACAAATTGAGTGAGTAGCATTC & 471 & 60 \\
\hline & Ex14 & ACCTGTCAACCTTTTTAAGAAC & CCAAGATCCAAGTAGGTCAAC & 445 & 60 \\
\hline
\end{tabular}

${ }^{\text {aExon }} 1$ of EXT1 and exon 2 of EXT2 were amplified in 3 and 2 fragments, respectively. Ta, annealing temperature.
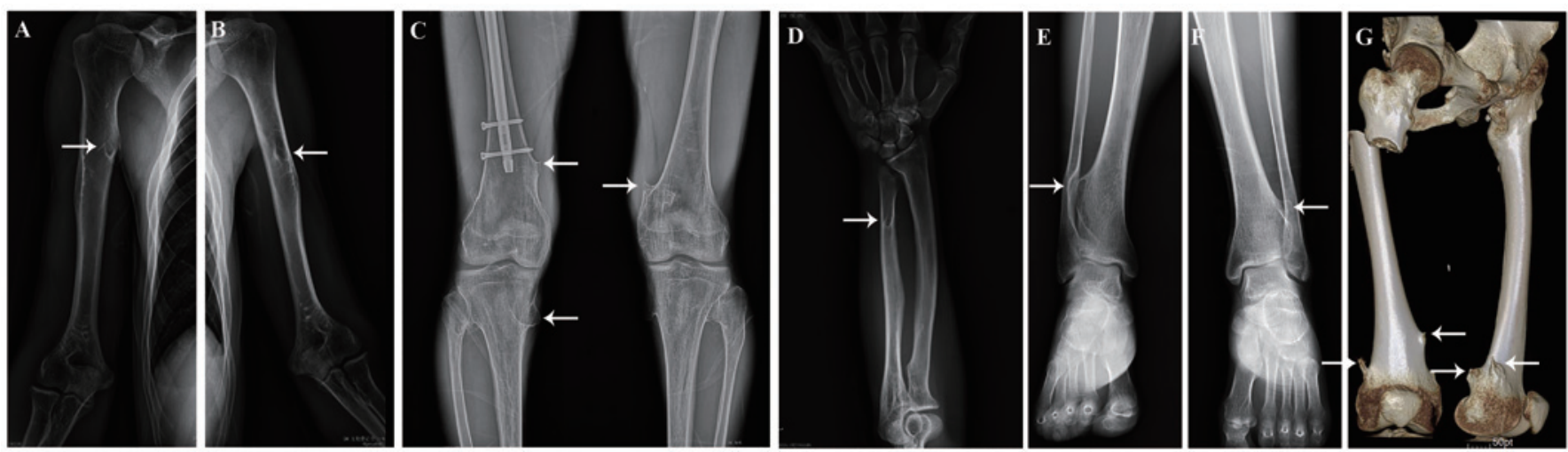

Figure 2. Posterior-anterior radiograph and 3D reconstruction images of osteochondromas (indicated by white arrows) in the proband. (A and B) Single osteochondroma in the bilateral humeri. (C) MOs in the femurs and fibula of the lower limbs. (D) Osteochondroma in the juxta-epiphyseal region of the left ulnar. (E and F) Osteochondromas in the bilateral tibiae. (G) 3D reconstruction images of knees of the MO proband. MO, multiple osteochondroma; 3D, three-dimensional.

flanking intronic regions of the EXT1 and EXT2 genes, no mutations were detected in the EXT1 gene, but a heterozygous deletion of two nucleotides (CT) at c.119_120 in exon 2 of the
EXT2 gene was found in the proband (Fig. 3). Subsequently, this variation was detected in all the 5 affected individuals (II-7, II-17, III-9, III-10 and III-18) in this pedigree by PCR and 
A

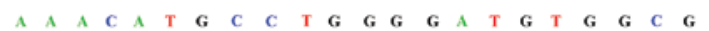

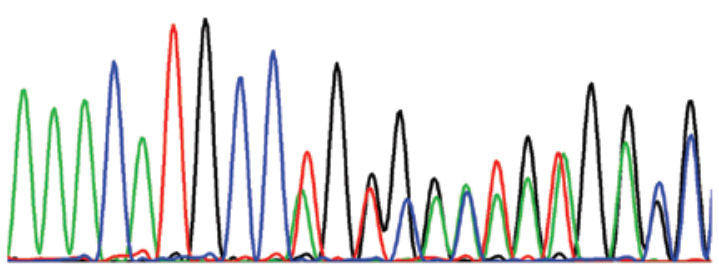

C

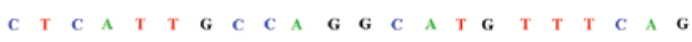

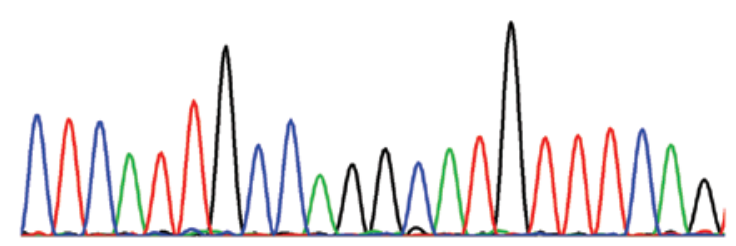

B

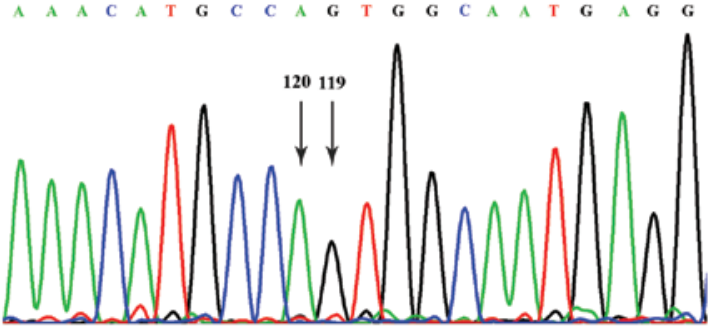

D

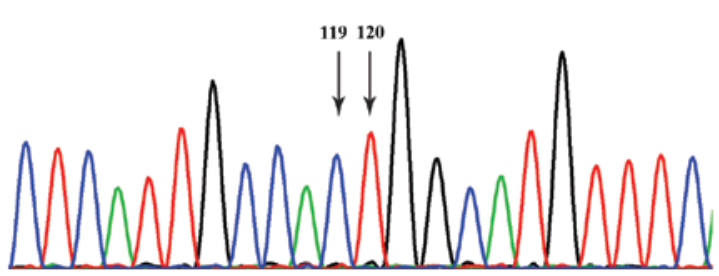

Figure 3. Sanger sequencing results from DNA of the proband's blood and from plasmids extracted from E. coli. (A) Reverse-sequencing result of the proband from DNA in blood. (B) Reverse-sequencing result of the unaffected family members and normal controls from DNA in blood. (C) Forward-sequencing result of the plasmid showing the deletion of two nucleotides (CT) at c.119_120 of the EXT2 gene. (D) Forward-sequencing result of normal EXT2 sequence from plasmid.

A

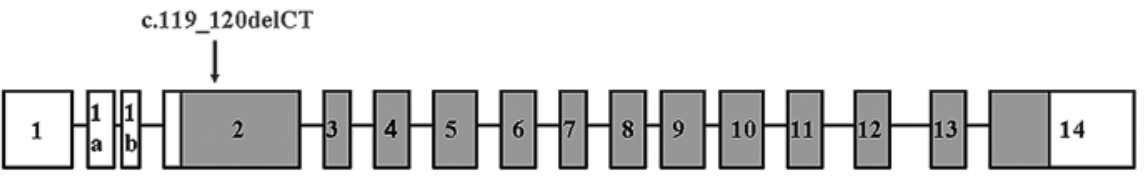

B Normal EXT2 protein structure

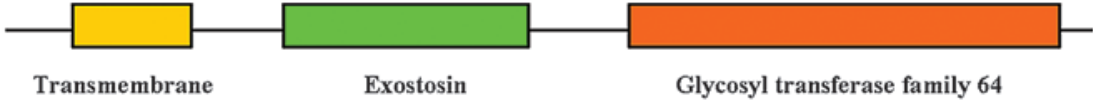

Mutated EXT2 protein structure

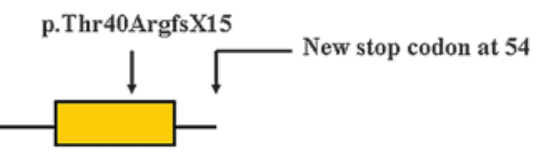

C

$\begin{array}{ll}\text { Homo sapiens } & \text { RIYYITLFSIVLLGLIATGMFQFWPHSIESSND } \\ \text { Pan troglodytes } & \text { RIYYITLFSIVLLGLIATGMFQFWPHSIESSND } \\ \text { Macaca mulatta } & \text { RIYYITLFSIVLLGLIATGMFQFWPHSIESSND } \\ \text { Canis lupus familiaris } & \text { RIYYITLFSIVLLGLIATGMFQFWPHSIESSSD } \\ \text { Mus musculus } & \text { RIYYVTLFSIVLLGLIATGMFQFWPHSIESSSD } \\ \text { Rattus norvegicus } & \text { RIYYVTLFSIVLLGLIATGMFQFWPHSIESSSD } \\ \text { Bos Taurus } & \text { RIYYITLFSIVLLGLIATGMFQFWPHSIESSGD } \\ \text { Gallus gallus } & \text { RIYYITLFSIVLLGLIATGVFQFWPHSIESSSD } \\ \text { Xenopus tropicalis } & \text { RVYYIALFSVVLLGLIATGMFQFWPHTIEPSTD } \\ \text { Danio rerio } & \text { RIYYITLFSVVLLGLIATGMFQFWPHSIESSAE } \\ & \text { * **::*** :******** ****** ** * : }\end{array}$

Figure 4.EXT2 gene and protein structural and homology study result of the identified c.119_120delCT (p.Thr40ArgfsX15) frameshift mutation. (A) Intron-exon structure of the EXT2 gene. (B) Comparison of the functional domains of EXT2 proteins encoded by mutated and normal EXT2 genes. (C) Multiple sequence alignment of EXT2 orthologs around codon 40. Codon 40 is highly conserved across various vertebrate species.

direct sequencing, and was not detected in any of the 4 unaffected family members (II-5, III-11, III-14 and III-17) or in the 50 healthy controls. Thus, this finding suggested that the novel c.119_120delCT frameshift mutation in the EXT2 gene was the causative mutation for MO in this family.
In silico analyses indicate the critical impact of the c.119_120delCT mutation on EXT2 function. The EXT2 gene consists of 16 exons, and the c.119_120delCT mutation was located in exon 2 (Fig. 4A). To understand the potential impact of the c.119_120delCT frameshift mutation on EXT2 
function, in silico analyses were performed and the predicted result was disease-causing. The EXT2 protein is composed of 718 amino acids, with one transmembrane region (amino acids 26-46) and two critical domains: The exostosin domain (amino acids 100-380) and the glycosyltransferase family 64 domain (amino acids 456-701) (Fig. 4B). The c.119_120delCT frameshift mutation results in the substitute of amino acid Threonine by Arginine at position 40 and creates a premature translational termination signal at amino acid position 54 of the EXT2 protein (Fig. 4B). Compared with normal EXT2 protein, the mutated EXT2 protein lacks 665 amino acids at the C-terminus, including the two critical exostosin and glycosyltransferase family 64 domains. Thr40 is located in the transmembrane region, which is highly conserved across various vertebrate species (Fig. 4C), indicating its functional importance.

\section{Discussion}

EXT1 and EXT2 are tumour suppressor genes encoding for endoplasmic reticulum-localized type II transmembrane glycoproteins (15). EXT1 and EXT2 form a hetero-oligomeric complex in the Golgi apparatus that catalyses the alternative transfer of $\mathrm{N}$-acetyl-glucosamine and D-glucuronic acid residues to the elongating HS glycosaminoglycan chain (16). This observation is consistent with the notion that inherited mutations in either of the two EXT genes could result in MO. HS and HS proteoglycans are known as regulators of the distribution and receptor binding of signalling molecule family members such as transforming growth factor- $\beta$, fibroblast growth factor, and Indian hedgehog (IHH) (17). Previously, studies showed that mutations in the EXT1/EXT2 genes impaired the biosynthesis of HS and that a decreased content of HS disturbs the $\mathrm{IHH} /$ parathyroid hormone-related peptide signalling pathway in MO; this disturbance leads to abnormal endochondral ossification resulting in exostoses formation $(18,19)$.

EXT1 and EXT2 gene mutations have been reported to be involved in the pathogenesis of $\mathrm{MO}$ and are responsible for $70-95 \%$ of MO cases. EXT1 accounts for $56-78 \%$ of the MO families, whereas an EXT2 mutation is detected in $21-44 \%$ of cases (20). EXT1/EXT2 mutation analysis demonstrated that mutations in EXT1 are more common in the Caucasian population in North America and Europe. However, these associations were different in the Chinese population, where mutations in EXT2 were more common than EXT1 mutations (21). In the past 10 years, 67 Chinese families affected with $\mathrm{MO}$ and 10 spontaneous cases affected with MO have been screened for mutations in EXT1 or EXT2. Mutations in the EXT1 gene account for $37.7 \%$ (29/77) of the patients, whereas EXT2 mutations account for $44.2 \%$ (34/77), which is higher than that of EXT1. Based on all the aforementioned data, EXT2 mutations may be responsible for more cases of MO in the Chinese population. EXT2 mutations tend to be concentrated in the first two-thirds of the coding region, and mutations may occur more commonly in exon 2 compared with other exons of EXT2, whereas mutations in EXT1 are reported to be scattered throughout the complete gene.

In the present study, a large family with MO was investigated and a sequence analysis was performed of the entire coding regions of EXT1 and EXT2 for the proband (III-16).
A heterozygous deletion of two nucleotides (CT) was found at c.119_120 in exon 2 of EXT2 in the proband, and this variation was also present in all the other 5 affected patients (II-7, II-17, III-9, III-10 and III-18) from this pedigree, but was not detected in any of the 4 unaffected family members (II-5, III-11, III-14 and III-17) or in the 50 healthy controls. This mutation (c.119_120delCT; p.Thr40ArgfsX15) results in a frameshift mutation starting from amino acid position 40 and introduces a premature termination signal at amino acid 54, which leads to the production of a truncated EXT2 protein with only 53 amino acids, 665 amino acids shorter than the normal EXT2 protein. Furthermore, the c.119_120delCT mutation was located in the transmembrane region of the EXT2 protein, the mutated EXT2 protein lacking 665 amino acids at the C-terminus, which included the two critical exostosin and glycosyltransferase family 64 domains.

In conclusion, the present study identified a novel deleterious frameshift mutation, c.119_120delCT (p.Thr40ArgfsX15), in the transmembrane region of the EXT2 gene from a large MO family. This study is useful for extending the known mutational spectrum of EXT2, for understanding the genetic basis of $\mathrm{MO}$ in the patients studied, and for further application of mutation screening in the genetic counseling and subsequent prenatal diagnosis of this family.

\section{Acknowledgements}

The authors would like to thank all the study participants for their interest and cooperation in this study.

\section{References}

1. Krooth RS, MacKlin MT and Hilbish TF: Diaphysial aclasis (multiple exostoses) on Guam. Am J Hum Genet 13: 340-347, 1961.

2. Hennekam RC: Hereditary multiple exostoses. J Med Genet 28: 262-266, 1991.

3. Schmale GA, Conrad EU III and Raskind WH: The natural history of hereditary multiple exostoses. J Bone Joint Surg Am 76: 986-992, 1994.

4. Bovée JV: Multiple osteochondromas. Orphanet J Rare Dis 3: 3, 2008.

5. Lüdecke HJ, Ahn J, Lin X, Hill A, Wagner MJ, Schomburg L, Horsthemke B and Wells DE: Genomic organization and promoter structure of the human EXT1 gene. Genomics 40: 351-354, 1997.

6. Clines GA, Ashley JA, Shah S and Lovett M: The structure of the human multiple exostoses 2 gene and characterization of homologs in mouse and Caenorhabditis elegans. Genome Res 7: 359-367, 1997.

7. Wise CA, Clines GA, Massa H, Trask BJ and Lovett M: Identification and localization of the gene for EXTL, a third member of the multiple exostoses gene family. Genome Res 7: 10-16, 1997.

8. Wuyts W, Van Hul W, Hendrickx J, Speleman F, Wauters J, De Boulle K, Van Roy N, Van Agtmael T, Bossuyt P and Willems PJ: Identification and characterization of a novel member of the EXT gene family, EXTL2. Eur J Hum Genet 5: 382-389, 1997.

9. Van Hul W, Wuyts W, Hendrickx J, Speleman F, Wauters J, De Boulle K, Van Roy N, Bossuyt P and Willems PJ: Identification of a third EXT-like gene (EXTL3) belonging to the EXT gene family. Genomics 47: 230-237, 1998.

10. Senay C, Lind T, Muguruma K, Tone Y, Kitagawa H, Sugahara K, Lidholt K, Lindahl U and Kusche-Gullberg M: The EXT1/EXT2 tumor suppressors: Catalytic activities and role in heparan sulfate biosynthesis. EMBO Rep 1: 282-286, 2000. 
11. Lonie L, Porter DE, Fraser M, Cole T, Wise C, Yates L, Wakeling E, Blair E, Morava E, Monaco AP and Ragoussis J: Determination of the mutation spectrum of the EXT1/EXT2 genes in British Caucasian patients with multiple osteochondromas, and exclusion of six candidate genes in EXT negative cases. Hum Mutat 27: 1160, 2006.

12. Ciavarella M, Coco M, Baorda F, Stanziale P, Chetta M, Bisceglia L, Palumbo P, Bengala M, Raiteri P, Silengo M, et al: 20 novel point mutations and one large deletion in EXT1 and EXT2 genes: Report of diagnostic screening in a large Italian cohort of patients affected by hereditary multiple exostosis. Gene 515: 339-348, 2013.

13. Delgado MA, Martinez-Domenech G, Sarrión P, Urreizti R, Zecchini L, Robledo HH, Segura F, de Kremer RD, Balcells S, Grinberg D and Asteggiano CG: A broad spectrum of genomic changes in latinamerican patients with EXT1/EXT2-CDG. Sci Rep 4: 6407, 2014.

14. Wuyts W and Van Hul W: Molecular basis of multiple exostoses: Mutations in the EXT1 and EXT2 genes. Hum Mutat 15: 220-227, 2000.

15. Lind T, Tufaro F, McCormick C, Lindahl U and Lidholt K: The putative tumor suppressors EXT1 and EXT2 are glycosyltransferases required for the biosynthesis of heparan sulfate. J Biol Chem 273: 26265-26268, 1998.
16. McCormick C, Duncan G, Goutsos KT and Tufaro F: The putative tumor suppressors EXT1 and EXT2 form a stable complex that accumulates in the Golgi apparatus and catalyzes the synthesis of heparan sulfate. Proc Natl Acad Sci USA 97: $668-673,2000$.

17. Esko JD and Lindahl U: Molecular diversity of heparan sulfate. J Clin Invest 108: 169-173, 2001.

18. Zak BM, Crawford BE and Esko JD: Hereditary multiple exostoses and heparan sulfate polymerization. Biochim Biophys Acta 1573: 346-355, 2002.

19. Koziel L, Kunath M, Kelly OG and Vortkamp A: Ext1-dependent heparan sulfate regulates the range of Ihh signaling during endochondral ossification. Dev Cell 6: 801-813, 2004

20. Jennes I, Pedrini E, Zuntini M, Mordenti M, Balkassmi S, Asteggiano CG, Casey B, Bakker B, Sangiorgi L and Wuyts W: Multiple osteochondromas: Mutation update and description of the multiple osteochondromas mutation database (MOdb). Hum Mutat 30: 1620-1627, 2009.

21. Xu L, Xia J, Jiang H, Zhou J, Li H, Wang D, Pan Q, Long Z, Fan C and Deng HX: Mutation analysis of hereditary multiple exostoses in the Chinese. Hum Genet 105: 45-50, 1999. 